\title{
A retrospective cohort study of risk factors and outcomes in older patients admitted to an inner-city geriatric unit in London during first peak of COVID-19 pandemic
}

\author{
Carmela Maniero ${ }^{1} \cdot$ Devan Patel $^{2} \cdot$ Asha Pavithran $^{2} \cdot{\text { Prasheena } \mathrm{Naran}^{2} \cdot \text { Fu Liang Ng }}^{1,3} \cdot$ John Prowle $^{4}$. \\ Dhanupriya Sivapathasuntharam ${ }^{2}(\mathbb{D}$
}

Received: 3 March 2021 / Accepted: 9 June 2021 / Published online: 6 July 2021

(c) Royal Academy of Medicine in Ireland 2021

\begin{abstract}
Purpose Compared to younger patients, coronavirus disease 2019 (COVID-19) clinical presentation in older people can be more heterogeneous and fatal. We aim to describe a cohort of older adults admitted in an inner-city London hospital during the first peak of the pandemic.

Methods A retrospective observational study that enrolled older adults consecutively admitted into two geriatric wards with suspected or confirmed severe acute respiratory syndrome coronavirus 2 (SARS-CoV-2) infection. We collected sociodemographic data, comorbidities, symptoms at presentation and/or during admission, biochemical and radiological data and outcomes at 28 days.

Results One hundred twenty-four patients were included, and 75\% were $>80$ years old. $19.5 \%$ of COVID-19 cases were judged to be hospital-acquired. More than half presented or developed typical symptoms, respiratory failure or fatigue. $46.8 \%$ were diagnosed with delirium, $24.2 \%$ with falls and dysphagia was present in $13.7 \%$.

The mortality rate was $29.8 \%$ and was higher among males, those $>80$ years, patients with a higher grade of frailty, a history of dementia or chronic kidney disease, as well as those diagnosed with respiratory failure, acute kidney injury or hypernatremia. Independent predictors of mortality were male sex, age $>80$ years, respiratory failure and hypernatremia.

Conclusion We have described a cohort of patients with SARS-CoV-2 infection in the first UK peak of the global pandemic. We found that these patients had significant frailty with multiple comorbidities. There was a high mortality and increased dependency and greater social care need in survivors.
\end{abstract}

Keywords Coronavirus pandemic $\cdot$ COVID-19 $\cdot$ Frailty $\cdot$ SARS-CoV-2

\section{Key summary points}

Aim: What are the clinical features of COVID-19 disease in older patients from a socially deprived area?

Findings: About half of patients had atypical presentation. Mortality rate was 30\%. Patients who survived had higher dependency and required increased social support.

Message: Covid-19 disease in older patients presents specific challenges for providing care, both in hospital and on discharge.

Dhanupriya Sivapathasuntharam

Dhanupriya.Sivapathasuntharam@nhs.net

1 Clinical Pharmacology Unit, QMUL, Barts NHS Trust, London, UK

2 Older People's Services, Royal London Hospital, Barts NHS Trust, London, UK

\section{Introduction}

COVID-19 is a heterogeneous disease. Typical symptoms are cough, pyrexia and dyspnoea; however, clinical manifestations can vary widely, from asymptomatic disease to pneumonia and life-threatening complications. In older people, clinical presentation can be more heterogeneous and subtle compared to younger patients, with delirium on presentation as common as $20-30 \%$ of cases [1-3].

3 Department of Clinical Pharmacology, St George's, University of London, London, UK

4 Critical Care and Perioperative Medicine Research Group, QMUL, Barts NHS Trust, London, UK 
Older patients have been found to have significantly higher mortality [4]. Other risk factors include male sex, hypertension and type 2 diabetes [5-7].

There is a strong correlation between Alzheimer's dementia and COVID-19 death rates. Whilst this may be multifactorial, it has been postulated the ApoE4 genotype, which is more commonly associated in dementia and delirium, may also have a specific role in increasing mortality [8].

The effects of severe acute respiratory syndrome coronavirus 2 (SARS-CoV-2) infection differ among ethnic groups. Recent meta-analyses have shown that Black, Hispanic and Asian populations present with higher rates of SARS-CoV-2 infection and mortality $[9,10]$. Possible causes include comorbidities, inequality in care access and different living arrangements.

The inner-city area served by our institution has distinctive characteristics compared to the rest of the UK, with significant levels of social deprivation: Tower Hamlets has a high population density, is ethnically diverse and ranked as the 10th most deprived out of 326 areas in England in terms of average level of deprivation and is the most income deprived in regard to older people [11, 12].

Therefore, we had the opportunity to describe the characteristics of this unique population of patients treated in our Older Peoples' Service during the first peak of the COVID19 pandemic in the UK.

\section{Methods}

We retrospectively collected socio-demographic data (including age, race, sex, social care needs, type of residence), comorbidities, symptoms at presentation and/or during admission, biochemical and radiological data and outcomes at 28 days in a cohort of 124 older adults consecutively admitted with suspected COVID-19 disease from the Emergency Department to the two older people's services wards at the Royal London Hospital between 15th March 2020 and the 30th April 2020, the first UK wave of the coronavirus pandemic. The criteria for admission to our unit were patients aged above the age of 65 years who had significant needs and comorbidities that would benefit from being under the care of a geriatrician.

This study was performed as a sub-population analysis within the ethnicity and outcomes in patients hospitalised with COVID-19 infection in East London (ethical) study [13], involving analysis of routinely collected patient data by members of the usual care team and approved by NHS England Health Research Authority (IRAS Project ID 283,512).

The cohort included patients above the age of 65 years of age with a positive swab (PCR-SARS-CoV-2) $(n=102)$ and/or high clinical suspicion of COVID-19 disease who were swab-negative $(n=22)$. Acute kidney injury (AKI) was diagnosed using KDIGO criteria [14]. Preadmission frailty was assessed on admission by the Emergency Department, and for this study retrospectively by two geriatricians using the Rockwood Clinical Frailty Scale (CFS) [15], a common and validated clinical assessment tool. The tool has recently been updated, but we have used the original criteria, as at the time of data collection, that was what was available. The scale ranges from very fit $(\mathrm{CFS}=1)$ to very severely frail $(\mathrm{CFS}=8)$ and terminally ill $(\mathrm{CFS}=9)$. Frailty was defined as CFS more than or equal to 5 .

A third geriatrician reviewed the discrepant scores. The CFS scores in this study refer to the specialist geriatrician assessment. Hospital-acquired COVID-19 disease was defined as an infection that is acquired in hospital by a patient who was admitted for a separate reason and with a prior negative test (at least 15 days prior to a positive COVID-19 diagnosis) [16]. We also considered hospitalacquired those readmitted with SARS-CoV-2 infection within 5 days of previous hospital discharge.

\section{Statistical analyses}

Differences between two independent groups were compared by t-tests for parametric distributions and by the Mann-Whitney test for non-parametric distributions. Differences between two dependent groups were compared by one-sample t-tests for parametric distributions. Differences in contingency tables were compared using chisquared and Fisher's exact tests, depending on expected numbers. Logistic regression was used to estimate the odds ratio for mortality. Due to the missing values for several haematological and biochemistry investigations, variables with more than $10 \%$ missing were excluded from the multivariate analysis. Statistical analyses were conducted using SPSS version 25 (IBM Corp). An alpha level of $\mathrm{P}<0.05$ was defined as statistically significant. Where appropriate, data is expressed as mean $\pm \mathrm{SD}$, or median (IQR).

\section{Results}

\section{Total cohort}

\section{Demographics and social history}

One hundred twenty-four patients were admitted during the period of observation. Their demographic characteristics are reported in Table 1. Of them, 50.8\% were males, their 
Table 1 Patients' demographic characteristics

\begin{tabular}{|c|c|c|c|c|}
\hline & All $(n=124)$ & Survived $(\mathrm{n}=87)$ & Died $(n=37)$ & $\mathrm{P}$ \\
\hline \multicolumn{5}{|l|}{ Demographics } \\
\hline Males, n (\%) & $63 / 124(50.8 \%)$ & $38 / 87(43 \%)$ & $25 / 37(67 \%)$ & 0.018 \\
\hline Age (median-IQR) & $83(79.25-89)$ & $83(78-89)$ & $84(81-88)$ & N.S \\
\hline Age $(\geq 80$ years $)$ & $93 / 124(75 \%)$ & $60 / 87(69 \%)$ & $33 / 37(89.2 \%)$ & 0.022 \\
\hline \multicolumn{5}{|l|}{ Ethnicity, n (\%) } \\
\hline White/White British & $80 / 124(64.6 \%)$ & $58 / 87(66.7 \%)$ & $23 / 36(63.9 \%)$ & N.S \\
\hline Asian/Asian British & $21 / 124(16.9 \%)$ & $12 / 87(13.8 \%)$ & $9 / 36(25.0 \%)$ & \\
\hline Black/Black British & $21 / 124(16.9 \%)$ & $17 / 87(19.5 \%)$ & $4 / 36(11.1 \%)$ & \\
\hline Data not available & $1 / 124(0.8 \%)$ & 0 & 1 & \\
\hline Hospital acquired & $24 / 124(19.4 \%)$ & $16 / 87(18.4 \%)$ & $8 / 37(21.6 \%)$ & N.S \\
\hline Non-hospital acquired & $100 / 124(80.6 \%)$ & $71 / 87(81.6 \%)$ & $29 / 37(78.4 \%)$ & \\
\hline \multicolumn{5}{|l|}{ Living situation, n (\%) } \\
\hline Own home & $93 / 124(75 \%)$ & $68 / 87(78.2 \%)$ & $25 / 37(67.6 \%)$ & 0.012 \\
\hline Sheltered accommodation & $11 / 124(8.9 \%)$ & $10 / 87(11.5 \%)$ & $1 / 37(2.7 \%)$ & \\
\hline Residential home & $7 / 124(5.6 \%)$ & $4 / 87(4.6 \%)$ & $3 / 37(8.1 \%)$ & \\
\hline Nursing home & $8 / 124(6.5 \%)$ & $2 / 87(2.3 \%)$ & $6 / 37(16.2 \%)$ & \\
\hline Psychiatric hospital & $4 / 124(3.2 \%)$ & $2 / 87(2.3 \%)$ & $2 / 37(5.4 \%)$ & \\
\hline Data not available & $1 / 124(0.8 \%)$ & $1 / 87(1.1 \%)$ & $0 / 37$ & \\
\hline \multicolumn{5}{|l|}{ Support, n (\%) } \\
\hline None & $48 / 102(47 \%)$ & $37 / 78(47.4 \%)$ & $11 / 24(45.8 \%)$ & N.S \\
\hline Up to TDS & $31 / 102(30.4 \%)$ & $23 / 78(29.5 \%)$ & $8 / 24(33.3 \%)$ & \\
\hline QDS & $23 / 102(22.5 \%)$ & $17 / 78(21.8 \%)$ & $6 / 24(25 \%)$ & \\
\hline CSF (median-IQR) & $6(4-6)$ & $6(4-6)$ & $6(5-7)$ & 0.02 \\
\hline 1 to 4 & $35 / 124(28.2 \%)$ & $31 / 87(35.6 \%)$ & $4 / 37(10.8 \%)$ & 0.01 \\
\hline 5 to 9 & $89 / 124(71.8 \%)$ & $56 / 87(64.6 \%)$ & $33 / 37(89.2 \%)$ & \\
\hline \multicolumn{5}{|l|}{ Comorbidities, n (\%) } \\
\hline Dementia & $46 / 124(37.1 \%)$ & $26 / 87(29.9 \%)$ & $20 / 37(54.1 \%)$ & 0.015 \\
\hline Diabetes & $46 / 124(37.1 \%)$ & $32 / 87(36.8 \%)$ & $14 / 37(37.8 \%)$ & N.S \\
\hline Hypertension & $85 / 124(68.5 \%)$ & $56 / 87(64.4 \%)$ & $29 / 37(78.7 \%)$ & N.S \\
\hline Chronic kidney disease & $27 / 124(21.8 \%)$ & $14 / 87(16.1 \%)$ & $13 / 37(35.1 \%)$ & 0.031 \\
\hline COPD/Asthma & $33 / 124(26.6 \%)$ & $24 / 87(27.6 \%)$ & $9 / 37(24.3 \%)$ & N.S \\
\hline Active cancer & $16 / 124(12.9 \%)$ & $11 / 87(12.6 \%)$ & $5 / 37(13.5 \%)$ & N.S \\
\hline Recurrent falls & $39 / 124(31.5 \%)$ & $24 / 87(27.6 \%)$ & $15 / 37(40.5 \%)$ & N.S \\
\hline Stroke & $25 / 124(20.2 \%)$ & $16 / 87(18.4 \%)$ & $9 / 37(24.3 \%)$ & N.S \\
\hline Ischemic heart disease & $26 / 124(21.0 \%)$ & $17 / 87(19.5 \%)$ & $9 / 37(24.3 \%)$ & N.S \\
\hline Heart failure & $19 / 124(15.3 \%)$ & 10/87 (11.5\%) & $9 / 37(24.3 \%)$ & N.S \\
\hline Dyslipidaemia & $50 / 124(40.3 \%)$ & $33 / 87(37.9 \%)$ & $17 / 37(45.9 \%)$ & N.S \\
\hline Atrial fibrillation & $29 / 124(23.4 \%)$ & $18 / 87(20.7 \%)$ & $11 / 37(29.7 \%)$ & N.S \\
\hline Obesity & $22 / 101(21.8 \%)$ & $14 / 87(16.1 \%)$ & $8 / 37(25.0 \%)$ & N.S \\
\hline Depression & $20 / 124(16.1 \%)$ & 15/87 (17.2\%) & $5 / 37(13.5 \%)$ & N.S \\
\hline Dysthyroidism & $16 / 124(12.9 \%)$ & $11 / 87(12.6 \%)$ & $5 / 37(13.5 \%)$ & N.S \\
\hline Previous VTE/PE & $8 / 124(6.5 \%)$ & $5 / 87(5.7 \%)$ & $3 / 37(8.1 \%)$ & N.S \\
\hline Learning disabilities & $6 / 124(4.8 \%)$ & $3 / 87(3.4 \%)$ & $3 / 37(8.1 \%)$ & N.S \\
\hline Schizophrenia & $10 / 124(8.1 \%)$ & $8 / 87(9.2 \%)$ & $2 / 37(5.4 \%)$ & N.S \\
\hline Parkinson's disease & $5 / 124(4.0 \%)$ & $3 / 87(3.4 \%)$ & $2 / 37(5.4 \%)$ & N.S \\
\hline Liver disease & $5 / 124(4.0 \%)$ & $4 / 87(4.6 \%)$ & $1 / 37(2.7 \%)$ & N.S \\
\hline
\end{tabular}

median age was 83 (IQR 79.25-89) years and 75\% of them were older than $80.19 .5 \%$ of SARS-CoV-2 infections were judged to be hospital-acquired.
With self-reported ethnicity, $65.5 \%$ were White British, $17.2 \%$ were Asian British, and a similar percentage was Black British. The majority (75\%) of patients were from their own 
home or from sheltered accommodation (8.9\%). Of these, $47 \%$ of patients did not have any formal carer support. $30.4 \%$ had carers visiting up to three times a day and $22.5 \%$ up to four times a day.

Of the whole cohort, 37/124 (29.8\%) died. Those who died were more likely to be male $(P=0.018)$, older than 80 years $(P=0.022)$ and to have a CFS score of more than or equal to $5(P=0.010)$. The median length of stay for those who survived was 14.1 days (8.9-19.8) (Table 1).

\section{Frailty and comorbidities}

The median CFS score was 6 (IQR 4-6), with the overall frailty prevalence of $76.6 \%$. Comorbidities are shown in Table 1 . As a reflection of the frailty of this cohort, $37.1 \%$ had a formal diagnosis of dementia, and recurrent falls (defined as 2 or more in the past year) were reported in $31.5 \%$. Patients who died had more frequently a history of dementia (54.1\% vs. $29.9 \%, P=0.015)$ or chronic kidney disease $(35.1 \%$ vs. $16.1 \%, P=0.031)$.

\section{Symptoms at presentation or during admission}

More than half of the patients presented or developed typical symptoms (fever in 55.3\%, cough in $48.4 \%$ and shortness of breath in $60.5 \%$ ). Fatigue was present in half cases. Respiratory failure was diagnosed in $53.2 \%$ of cases (Table 2). On admission, $29.8 \%$ were diagnosed with AKI, $13.7 \%$ with hyponatremia and $17.7 \%$ with hypernatremia. $46.8 \%$ were diagnosed with delirium, $24.2 \%$ with falls and dysphagia (not related to a previous condition or new stroke) was present in $13.7 \%$ of patients.

Patients who died were more likely to have been diagnosed with respiratory failure (73\% vs. $44.8 \%, P=0.006$ ), AKI ( $48.6 \%$ vs. $21.8 \%, P=0.005)$ or hypernatremia $(32.4 \%$ vs. $11.5 \%, P=0.009)$.
Table 2 Clinical presentation, haematological, biochemical and radiological characteristics of the patients (whole cohort, survived and died)

\begin{tabular}{|c|c|c|c|c|}
\hline & All (total $n=124)$ & Survived $(n=87)$ & Died $(n=37)$ & $P$ \\
\hline \multicolumn{5}{|l|}{ Clinical presentation } \\
\hline Fever & $66 / 124(53.2 \%)$ & $43 / 87(49.4 \%)$ & $23 / 37(62.2 \%)$ & N.S \\
\hline Cough & $60 / 124(48.4 \%)$ & $42 / 87(48.3 \%)$ & $18 / 37(48.6 \%)$ & N.S \\
\hline Shortness of breath & $75 / 124(60.5 \%)$ & $48 / 87(55.2 \%)$ & $27 / 37(73.0 \%)$ & N.S \\
\hline Delirium & $58 / 124(46.8 \%)$ & $44 / 87(50.6 \%)$ & $14 / 37(37.8 \%)$ & N.S \\
\hline Vomiting & $12 / 124(9.7 \%)$ & $6 / 87(6.9 \%)$ & $6 / 37(16.2 \%)$ & N.S \\
\hline Fall & $30 / 124(24.2 \%)$ & $22 / 87(25.3 \%)$ & $8 / 37(21.6 \%)$ & N.S \\
\hline Fatigue & $62 / 124(50 \%)$ & $44 / 87(50.6 \%)$ & $21 / 37(56.8 \%)$ & N.S \\
\hline Diarrhoea & $15 / 124(12.1 \%)$ & $12 / 87(13.8 \%)$ & $3 / 37(8.1 \%)$ & N.S \\
\hline Acute kidney injury & $37 / 124(29.8 \%)$ & $19 / 87(21.8 \%)$ & $18 / 37(48.6 \%)$ & 0.005 \\
\hline Respiratory failure & $66 / 124(53.2 \%)$ & $39 / 87(44.8 \%)$ & $27 / 37(73.0 \%)$ & 0.006 \\
\hline Dysphagia & $17 / 124(13.7 \%)$ & $10 / 87(11.5 \%)$ & $7 / 37(18.9 \%)$ & N.S \\
\hline \multicolumn{5}{|c|}{ Haematology and biochemistry } \\
\hline Hyponatremia & $17 / 124(13.7 \%)$ & $10 / 87(11.5 \%)$ & $7 / 37(18.9 \%)$ & N.S \\
\hline Hypernatremia & $22 / 124(17.7 \%)$ & $10 / 87(11.5 \%)$ & $12 / 37(32.4 \%)$ & 0.009 \\
\hline Low lymphocytes & $71 / 124(57.3 \%)$ & $48 / 87(55.2 \%)$ & $23 / 37(62.2 \%)$ & N.S \\
\hline Elevated D-dimer & $71 / 90(78.9 \%)$ & $48 / 63(76.2 \%)$ & $23 / 27(85.2 \%)$ & N.S \\
\hline Elevated CRP & $115 / 124(92.7 \%)$ & 78/87(89.7\%) & $37 / 37(100 \%)$ & N.S \\
\hline Elevated creatine kinase & $32 / 78(41 \%)$ & $19(37.3 \%)$ & $13 / 27(48.1 \%)$ & N.S \\
\hline Elevated troponin $\mathrm{T}$ & $61 / 94(64.9 \%)$ & $37(57.8 \%)$ & $24 / 30(80.0 \%)$ & 0.04 \\
\hline Elevated ALT & $26 / 117(21.1 \%)$ & $13 / 82(15.9 \%)$ & $13 / 35(37.1 \%)$ & 0.016 \\
\hline Elevated ALP & $26 / 123(22.2 \%)$ & $12 / 86(14 \%)$ & $14 / 37(37.8 \%)$ & 0.007 \\
\hline Elevated LDH & $56 / 72(77.8 \%)$ & $38 / 51(74.5 \%)$ & $18 / 21(85.7 \%)$ & N.S \\
\hline Elevated ferritin & $61 / 99(61.6 \%)$ & $43 / 67(64.2 \%)$ & $18 / 32(56.3 \%)$ & N.S \\
\hline \multicolumn{5}{|l|}{ CXR findings } \\
\hline Normal & $37 / 120(30.8 \%)$ & $31 / 86(36.0 \%)$ & $6 / 34(17.6 \%)$ & N.S \\
\hline Unilateral changes & $31 / 120(25.8 \%)$ & $24 / 86(27.9 \%)$ & $7 / 34(20.6 \%)$ & \\
\hline Bilateral changes & $41 / 120(34.2 \%)$ & $25 / 86(29.1 \%)$ & $16 / 34(47.1 \%)$ & \\
\hline Other & $11 / 120(9.2 \%)$ & $6 / 86(7 \%)$ & $5 / 34(14.7 \%)$ & \\
\hline
\end{tabular}

N.S. non-significant, $C R P$ c-reactive protein, $A L T$ alanine aminotransferase, $A L P$ alkaline phosphatase, $L D H$ lactate dehydrogenase 


\section{Diagnostics}

$57.3 \%$ of patients presented with lymphocytopenia, $78.9 \%$ with raised D-dimer and $92.7 \%$ with raised C-reactive protein. $30.8 \%$ of patients had normal chest radiography. Unilateral and bilateral changes were found in $25.8 \%$ and $34.2 \%$ of patients, respectively. Patients who died had higher troponin, alanine transaminase (ALT) and alkaline phosphatase (ALP) levels (Table 2).

\section{Multivariate analysis}

On multivariate analysis, the variables remaining as an independent predictor of mortality were male sex, age $>80$ years, presentation with respiratory failure and hypernatremia (Fig. 1).

\section{SARS-CoV-2 swab-positive patients vs. swab-negative patients with suspected disease}

We also compared patients with a positive swab (PCRSARS-CoV-2) $(n=102)$ with those with high clinical suspicion (based on history, examination, radiographical changes and/or laboratory findings) of COVID-19 disease who had a negative swab $(n=22)$. In terms of demographics, the two groups were comparable with regards to sex, ethnicity, residence prior to admission, care needs and clinical frailty scores. The swab-negative group was however older (median 91 years (IQR 84.5-93) vs. 82.5 years $(78-88), P=0.002)$. There were no statistical differences between the groups with regards to comorbidities, and the only differences in admission symptoms were that the swab-positive group was more likely to present with fatigue (56.9\% vs. $31.8 \%$, $P=0.037)$ and hypernatremia $(21.6 \%$ vs. $0 \%, P=0.013)$. When comparing the proportions of patients with abnormal
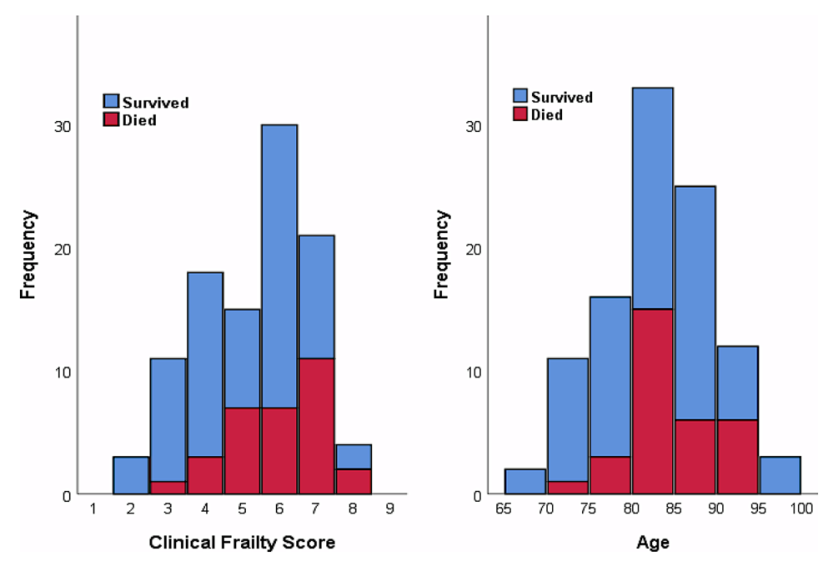

Fig. 1 Histograms show frequency of patients who died (red) and survived (blue), with bins for Clinical Frailty Score (left) and age (right) haematological, biochemistry and chest radiograph results, there were no differences between the groups.

Whilst there were no differences in the mortality rate (30.4\% in swab-positive group vs. $27.3 \%$ ), the patients who were swab-positive for PCR-SARS-CoV-2 were more likely to suffer functional decline requiring increased support at home (32.2\% vs. $0 \%, P=0.008)$. These results are available in full in Table 3.

\section{SARS-CoV-2 PCR-positive sub-group analysis}

To assess whether the overall study results were influenced by the minority group of PCR-SARS-CoV-2-negative patients, we followed this with a sensitivity analysis of the subgroup of patients who were confirmed to be PCR-SARSCoV-2-positive (Table 4) $(n=102$, Supplemental Tables 1 and 2). This is similar to the findings as the overall cohort, where demographics associated with death were male $(\mathrm{P}=0.031)$ being older than 80 years $(\mathrm{P}=0.022)$, living in a residential or nursing home pre-admission $(\mathrm{P}=0.012)$ and having higher frailty scores $(\mathrm{P}=0.008)$ and the presence of dementia $(\mathrm{P}<0.001)$. Patients who died presented more frequently with $\operatorname{AKI}(\mathrm{P}=0.005)$, respiratory failure $(\mathrm{P}=0.016)$ and hypernatremia $(\mathrm{P}=0.005)$. With regards to biochemistry and radiological investigations, SARS-CoV-2 swab-positive patients who died were more likely to have elevated $\mathrm{D}$-dimer $(P=0.027)$, elevated alanine transaminase $(P=0.049)$ and alkaline phosphatase $(\mathrm{P}=0.002)$ and to have an abnormal chest radiographs $(\mathrm{P}=0.021)$.

\section{Discussion}

This study describes the characteristics of the patients admitted to our Older People's Services wards in an inner-city Central London Teaching Hospital during the first wave of the global coronavirus pandemic of 2020. Although more than half did present with classic symptoms, many were notable for the absence of respiratory symptoms, but

Table 3 Logistic regression analysis estimating odds ratio for mortality

\begin{tabular}{lrlll}
\hline & B & S.E & Sig & $\operatorname{Exp}(\mathrm{B})$ \\
\hline Male & 1.106 & 0.553 & 0.045 & 3.023 \\
Age $>80$ & 1.441 & 0.694 & 0.038 & 4.226 \\
Resp failure & 1.061 & 0.538 & 0.049 & 2.889 \\
HyperNa & 2.038 & 0.553 & 0.000 & 7.679 \\
Constant & -4.061 & 0.902 & 0.000 & 0.017 \\
\hline
\end{tabular}

Due to the missing values for several haematological and biochemistry investigations, variables with more than $10 \%$ missing were excluded from the multivariate analysis. Ninety-eight individuals ( $29.6 \%$ died) had the complete dataset for this analysis 
Table 4 Clinical presentation, haematological, biochemical and radiological characteristics of the patients (positive PCR-swab vs. negative PCRswab)

\begin{tabular}{|c|c|c|c|c|c|c|}
\hline & & $\begin{array}{l}\text { All positive swabs } \\
(n=102)\end{array}$ & $\begin{array}{l}\text { All negative swabs } \\
(n=22)\end{array}$ & & & $P$ \\
\hline \multicolumn{7}{|l|}{ Clinical presentation } \\
\hline Fever & & $56 / 102(54.9 \%)$ & $10 / 22(45.5 \%)$ & & & N.S \\
\hline Cough & & $51 / 102(50.0 \%)$ & $9 / 22(40.9 \%)$ & & & N.S \\
\hline Shortness of breath & & $61 / 102(59.8 \%)$ & $14 / 22(63.6 \%)$ & & & N.S \\
\hline Delirium & & $52 / 102(51.0 \%)$ & $6 / 22(27.3 \%)$ & & & N.S \\
\hline Vomiting & & $11 / 102(10.8 \%)$ & $1 / 22(4.5 \%)$ & & & N.S \\
\hline Fall & & $22 / 102(21.6 \%)$ & $8 / 22(36.4 \%)$ & & & N.S \\
\hline Fatigue & & $58 / 102(56.9 \%)$ & $7 / 22(31.8 \%)$ & & & 0.037 \\
\hline Diarrhoea & & $14 / 102(13.7 \%)$ & $1 / 22(4.5 \%)$ & & & N.S \\
\hline Acute Kidney Injury & & $31 / 102(30.4 \%)$ & $6 / 22(27.3 \%)$ & & & N.S \\
\hline Respiratory Failure & & $54 / 102(52.9 \%)$ & $12 / 22(54.5 \%)$ & & & N.S \\
\hline Dysphagia & & $15 / 102(14.7 \%)$ & $2 / 22(9.1 \%)$ & & & N.S \\
\hline \multicolumn{7}{|l|}{ Haematology and biochemistry } \\
\hline Hyponatremia & & $15 / 102(14.7 \%)$ & $2 / 22(9.1 \%)$ & & & N.S \\
\hline Hypernatremia & & $22 / 102(21.6 \%)$ & $0(0 \%)$ & & & 0.013 \\
\hline Low lymphocytes & & $59 / 102(57.8 \%)$ & $12 / 22(54.5 \%)$ & & & N.S \\
\hline Elevated D-dimer & & $66 / 76(86.8 \%)$ & $12 / 14(85.7 \%)$ & & & N.S \\
\hline Elevated CRP & & $97 / 102(95.1 \%)$ & $18 / 22(81.8 \%)$ & & & N.S \\
\hline Elevated creatine kinase & & $27 / 66(40.9 \%)$ & $5 / 12(41.7 \%)$ & & & N.S \\
\hline Elevated troponin $\mathrm{T}$ & & $48 / 79(60.8 \%)$ & $13 / 15(86.7 \%)$ & & & N.S \\
\hline Elevated ALT & & $24 / 97(24.7 \%)$ & $2 / 20(10 \%)$ & & & N.S \\
\hline Elevated ALP & & $18 / 101(17.8 \%)$ & $8 / 22(36.4 \%)$ & & & N.S \\
\hline Elevated LDH & & $47 / 62(75.8 \%)$ & $9 / 10(90 \%)$ & & & N.S \\
\hline Elevated ferritin & & $54 / 83(65.1 \%)$ & $7 / 16(43.8 \%)$ & & & N.S \\
\hline \multicolumn{7}{|l|}{ CXR findings } \\
\hline Normal & $31 / 99(31.3 \%)$ & & & $6 / 21(28.6 \%)$ & N.S & \\
\hline Unilateral changes & $26 / 99(26.3 \%)$ & & & $5 / 21(23.8 \%)$ & & \\
\hline Bilateral changes & $34 / 99(34.3 \%)$ & & & $7 / 21(33.3 \%)$ & & \\
\hline Other & $8 / 99(8.1 \%)$ & & & $3 / 21(14.3 \%)$ & & \\
\hline \multicolumn{7}{|l|}{ Outcomes } \\
\hline Deaths & & $31 / 102(30.4 \%)$ & $6 / 22(27.3 \%)$ & & & N.S \\
\hline Increased support at home & & $19 / 59(32.2 \%)$ & $0 / 15(0 \%)$ & & & 0.008 \\
\hline Change in discharge destination & & $8 / 59(13.6 \%)$ & $1 / 15(6.7 \%)$ & & & N.S \\
\hline
\end{tabular}

N.S. non-significant, $C R P$ c-reactive protein, $A L T$ alanine aminotransferase, $A L P$ alkaline phosphatase, $L D H$ lactate dehydrogenase

presenting instead with vomiting and diarrhoea, as well as delirium and falls, which have been previously described [17]. Of interest, a third of the patients had a normal chest radiograph, and almost half did not meet the definition of respiratory failure. This constellation of symptoms and signs emphasises the need to recognise the common non-respiratory presentations of SARS-CoV-2 in the older patient and that 'atypical' presentations may in fact be more 'typical' for this cohort.

Our cohort had a higher prevalence of frailty (CFS 5-9) at $71.8 \%$, compared to that reported in the recent COPE study $(49.4 \%)$ [18]. The overall mortality in our cohort was close to $30 \%$ and comparable to other studies despite the burden of frailty present, multiple comorbidities and a significant prevalence of 'geriatric syndromes'. One of the reasons of mitigated mortality may be attributed to the fact that they were admitted in a dedicated unit with specialist geriatricians and nursing staff with skills in managing frailty, dementia and delirium. We should also consider that those admitted to a hospital setting represent a selected subset of the older population, and these figures do not account for mortality in the community.

As expected, patients who survived had a greater degree of dependency, with nearly one-fifth of patients needing 
more support at home and $10 \%$ needing a new nursing home placement on discharge. This may correlate with the medium and long-term impact of the pandemic on the support care system and the economic resources required to sustain care of those older, frail patients who survive.

Our study showed a high prevalence of patients from Black, Asian and minority ethnic (BAME) backgrounds, reflecting the local general population. However, unlike the previously published studies on BAME patients [13, 19], we were not able to demonstrate any significant differences in outcomes according to ethnicity in our cohort.

We have demonstrated that dementia and CKD are both risk factors for mortality, similar to other studies [8, 19, 20]. AKI has been found to be common in SARS-CoV-2 infection, mainly in older people [21]. Patients with COVID-19 who present or develop AKI have higher mortality and morbidity, prolonged hospital stay and higher probability to be admitted in intensive care [22]. Direct nephrotoxic effects of SARS-COV-2 infection have been described [23] and mediated by endothelial injury, thrombotic events and glomerulopathy [24-26]. Older patients may be more vulnerable to AKI due ageing kidneys and pre-existing comorbidities [27], sepsis, hypovolemia and dehydration.

Hypernatremia is also associated with a higher mortality. This has been described previously in younger patients in intensive care [28]. Factors that have been thought to contribute to it include insensible losses from persistent pyrexia and increased respiration rate as well as management strategies during that period when more conservative fluid regimes were adopted [29]. In frailer older patients with dementia and delirium, preventing and managing hypernatremia can be particularly challenging even in usual circumstances. Maintaining adequate oral nutrition and hydration requires skilled nursing and a patient-centred approach, requiring time and patience. We found that $13.7 \%$ of our patients developed dysphagia (not thought to be due to a new stroke). Dysphagia can occur in patients with compromised respiratory function, due to incoordination between swallowing and respiration [30]. General deconditioning and fatigue may further exacerbate dysphagia together with preexisting age-related decline in swallowing. Dysphagia is likely to further amplify fluid and nutritional deficiencies.

The pandemic and restrictions on visiting have been disproportionately detrimental to cognitively impaired patients, who are often more receptive to offers of oral nutrition and fluid from family members or familiar caregivers. Personal protective equipment poses an additional challenge in nursing this cohort of patients, as the unfamiliarity can seem alarming and frightening. Language barriers and lack of access to translation services during the pandemic represent further obstacles to communication, in the context of different ethnic backgrounds. All of these factors are likely to compound the difficulties of maintaining oral hydration and nutrition, as well as worsening or perpetuating delirium and associated challenging behaviour.

Our data demonstrated that the majority of patients were admitted from their own homes or sheltered accommodation, but nearly half of them were reported as not having any formal carers. However, anecdotally, we are aware that much care for older persons in our hospital catchment area is provided by family members and may result in the underrepresentation of the true level of care needs.

Five of our patients came from psychiatric facilities. 8.1\% of all our patients had a diagnosis of schizophrenia, and $4.8 \%$ had a confirmed learning disability. This is a particularly vulnerable subgroup of patients as they present due to difficulties in communicating their symptoms, maintaining physical distancing and hand hygiene [31, 32]. This is also the case in patients with dementia, particularly those that acquired SARS-CoV-2 infection nosocomially.

The comparison of PCR-positive against those who were PCR-negative but had a high clinical suspicion for COVID19 disease found that the former group was significantly younger. The only other relevant difference was a higher prevalence of reported fatigue and hypernatremia in the PCR-positive group. Of note, the mortality rates for these two subgroups were similar. These results should be interpreted with caution due to the small sample size of the swabnegative/high clinical suspicion group. Moreover, there are practical issues associated with acquiring swabs in older, frailer and confused patients who may be agitated and or have challenging behaviour, thus limiting appropriate sampling. The unfamiliarity of PPE and lack of familiar caregivers also play a role here too as barriers to cooperation for an intrusive test. These factors highlight the possibility of false-negatives in the older person cohort.

The main limitations of our study were the retrospective nature and the small size. It is also a single-centre study of a specific population of patients. This study was conducted during a time when our understanding of the disease was rapidly evolving including changing management strategies. Outcomes will be inevitably be affected by this.

\section{Conclusions}

In our study, we have been able to describe our experience of COVID-19 disease in older patients during the first UK peak of the global pandemic. Our population has characteristics similar to previously described cohorts of older people as well as some unique attributes. There is a high degree of frailty and comorbidities, resulting in increased mortality and increased dependency leading to higher care requirements in survivors. Dementia, CKD and frailty were associated with mortality as are AKI, hypernatremia and respiratory failure. We have also highlighted some of the 
practical challenges of diagnosing COVID 19 and caring for this complex group of patients during this time.

Supplementary information The online version contains supplementary material available at https://doi.org/10.1007/s11845-021-02679-z.

Availability of data and material Data are available upon request to the principal investigator and corresponding author.

\section{Declarations}

Ethics approval This is a sub-analysis of the ethical study which was reviewed by the Yorkshire \& The Humber - Bradford Leeds Research Ethics Committee and approved as anonymised analysis of routinely collected patient data without the need for consent by NHS England Health Research Authority (IRAS Project ID 283,512).

Conflict of interest The authors declare no competing interests.

\section{References}

1. Kennedy M, Helfand BKI, Gou RY et al (2020) Delirium in older patients With COVID-19 presenting to the emergency department. JAMA Netw open. Epub ahead of print. https://doi.org/10.1001/ jamanetworkopen.2020.29540

2. O'Hanlon S, Inouye SK (2020) Delirium: a missing piece in the COVID-19 pandemic puzzle. Age and Ageing. Epub ahead of print. https://doi.org/10.1093/ageing/afaa094

3. Zazzara MB, Penfold RS, Roberts AL et al (2021) Probable delirium is a presenting symptom of COVID-19 in frail, older adults: a cohort study of 322 hospitalised and 535 community-based older adults. Age Ageing. Epub ahead of print. https://doi.org/10.1093/ ageing/afaa223

4. Wu Z, McGoogan JM (2020) Characteristics of and Important Lessons From the Coronavirus Disease 2019 (COVID-19) Outbreak in China. JAMA. Epub ahead of print. https://doi.org/10. 1001/jama.2020.2648

5. Garnier-Crussard A, Forestier E, Gilbert T et al (2020) Novel coronavirus (COVID-19) epidemic: what are the risks for older patients? J Am Geriatr Soc. Epub ahead of print. https://doi.org/ 10.1111/jgs.16407

6. Du RH, Liang LR, Yang CQ et al (2020) Predictors of mortality for patients with COVID-19 pneumonia caused by SARSCoV-2: a prospective cohort study. Eur Respir J. Epub ahead of print. https://doi.org/10.1183/13993003.00524-2020

7. Zhou F, Yu T, Du R et al (2020) Clinical course and risk factors for mortality of adult inpatients with COVID-19 in Wuhan, China: a retrospective cohort study. Lancet. Epub ahead of print. https:// doi.org/10.1016/S0140-6736(20)30566-3

8. Kuo CL, Pilling LC, Atkins JL et al (2020) APOE e4 Genotype predicts severe COVID-19 in the UK Biobank Community Cohort. J Gerontol A Biol Sci Med Sci. Epub ahead of print. https://doi.org/10.1093/gerona/glaa131

9. Sze S, Pan D, Nevill CR et al (2020) Ethnicity and clinical outcomes in COVID-19: a systematic review and meta-analysis. EClinicalMedicine. Epub ahead of print. https://doi.org/10.1016/j. eclinm.2020.100630

10. Mackey K, Ayers CK, Kondo KK et al (2020) Racial and ethnic disparities in COVID-19-related infections, hospitalizations, and deaths. Ann Intern Med. Epub ahead of print. https://doi.org/10. 7326/m20-6306
11. Tower Hamlets Borough profile https://www.towerhamlets. gov.uk/Documents/Borough_statistics/Research-briefings/ Population_2_BP2018.pdf

12. The Ministry of Housing $C$ and LG. The english indices of deprivation (2019). (IoD2019). https://assets.publishing.service.gov. uk/government/uploads/system/uploads/attachment_data/file/ 835115/IoD2019_Statistical_Release.pdf

13. Apea VJ, Wan YI, Dhairyawan R et al (2021) Ethnicity and outcomes in patients hospitalised with COVID-19 infection in East London: an observational cohort study. BMJ Open 11: e042140

14. Kellum JA, Lameire N, Aspelin P et al (2012) Kidney disease: Improving global outcomes (KDIGO) acute kidney injury work group. KDIGO clinical practice guideline for acute kidney injury. Kidney International Supplements. Epub ahead of print. https:// doi.org/10.1038/kisup.2012

15. Rockwood K, Song X, MacKnight C et al (2005) A global clinical measure of fitness and frailty in elderly people. CMAJ. Epub ahead of print. https://doi.org/10.1503/cmaj.050051

16. Carter B, Collins JT, Barlow-Pay F et al (2020) Nosocomial COVID-19 infection: examining the risk of mortality. The COPENosocomial Study (COVID in Older PEople). J Hosp Infect. Epub ahead of print. https://doi.org/10.1016/j.jhin.2020.07.013

17. Gan JM, Kho J, Akhunbay-Fudge M et al (2020) Atypical presentation of COVID-19 in hospitalised older adults. Ir J Med Sci. Epub ahead of print. https://doi.org/10.1007/s11845-020-02372-7

18. Hewitt J, Carter B, Vilches-Moraga A et al (2020) The effect of frailty on survival in patients with COVID-19 (COPE): a multicentre, European, observational cohort study. Lancet Public Heal. Epub ahead of print. https://doi.org/10.1016/S2468-2667(20) 30146-8

19. Williamson EJ, Walker AJ, Bhaskaran K et al (2020) Factors associated with COVID-19-related death using OpenSAFELY. Nature. Epub ahead of print. https://doi.org/10.1038/s41586-020-2521-4

20. Hariyanto TI, Putri C, Situmeang RFV et al (2020) Dementia is a predictor for mortality outcome from coronavirus disease 2019 (COVID-19) infection. Eur Arch Psychiatry Clin Neurosci. Epub ahead of print. https://doi.org/10.1007/s00406-020-01205-z

21. Lin L, Wang X, Ren J et al (2020) Risk factors and prognosis for COVID-19-induced acute kidney injury: a meta-analysis. BMJ Open 10:e042573

22. Hamilton P, Hanumapura P, Castelino L et al (2020) Characteristics and outcomes of hospitalised patients with acute kidney injury and COVID-19. PLoS One. Epub ahead of print. https://doi.org/ 10.1371/journal.pone.0241544

23. Yalameha B, Roshan B, Bhaskar LVKS et al (2020) Perspectives on the relationship of renal disease and coronavirus disease 2019. J Nephropharmacology. Epub ahead of print. https://doi.org/10. 34172/npj.2020.22

24. Su H, Yang M, Wan C et al (2020) Renal histopathological analysis of 26 postmortem findings of patients with COVID-19 in China. Kidney Int. Epub ahead of print. https://doi.org/10.1016/j. kint.2020.04.003

25. Batlle D, Soler MJ, Sparks MA et al (2020) COVID-19 and ACE2 in Cardiovascular, Lung, and Kidney WorkingGroup. Acute Kidney Injury in COVID-19: Emerging Evidence of a Distinct Pathophysiology. J Am Soc Nephrol 31(7):1380-1383. https://doi.org/ 10.1681/ASN.2020040419. Epub 2020 May 4. PMID: 32366514; PMCID: PMC7350999

26. Ronco C, Reis T (2020) Kidney involvement in COVID-19 and rationale for extracorporeal therapies. Nature Reviews Nephrology. Epub ahead of print. https://doi.org/10.1038/ s41581-020-0284-7

27. Abdel-Kader K, Palevsky PM (2009) Acute kidney injury in the elderly. Clin Geriatr Med. Epub ahead of print. https://doi.org/10. 1016/j.cger.2009.04.001 
28. Redant S, Vanderhulst J, Maillart E et al (2020) Significance of Hypernatremia Due to SARS-CoV-2 Associated ARDSin Critically Ill Patients. J Transl Int Med 8(4):255-260. https://doi. org/10.2478/jtim-2020-0038. PMID: 33511052; PMCID: PMC7805286

29. Christ-Crain M, Hoorn EJ, Sherlock M et al (2020) Endocrinology in the Time of COVID-19: Management of diabetes insipidus and hyponatraemia. Eur J Endocrinol. Epub ahead of print. https://doi. org/10.1530/EJE-20-0338.

30. Mohan R, Mohapatra B (2020) Shedding light on dysphagia associated with COVID-19: the what and why. OTO Open. Epub ahead of print. https://doi.org/10.1177/2473974x20934770
31. Benson NM, Öngür D, Hsu J (2020) COVID-19 testing and patients in mental health facilities. Lancet Psychiat. Epub ahead of print. https://doi.org/10.1016/S2215-0366(20)30198-X

32. Zhu Y, Chen L, Ji H et al (2020) The risk and prevention of novel coronavirus pneumonia infections among inpatients in psychiatric hospitals. Neurosci Bull. Epub ahead of print. https://doi.org/10. 1007/s12264-020-00476-9

Publisher's Note Springer Nature remains neutral with regard to jurisdictional claims in published maps and institutional affiliations. 